\title{
BMJ Open Developing a theory-informed complex intervention to improve nurse-patient therapeutic engagement employing Experience-based Co-design and the Behaviour Change Wheel: an acute mental health ward case study
}

\author{
Sarah McAllister (D) , ${ }^{1}$ Alan Simpson,,${ }^{1}$ Vicki Tsianakas, ${ }^{1}$ Nick Canham, ${ }^{2}$ \\ Vittoria De Meo, ${ }^{2,3}$ Cady Stone, ${ }^{2}$ Glenn Robert (D) ${ }^{1}$
}

To cite: McAllister S,

Simpson A, Tsianakas V, et al. Developing a theory-informed complex intervention to improve nurse-patient therapeutic engagement employing Experience-based $\mathrm{Co}$-design and the Behaviour Change Wheel: an acute mental health ward case study. BMJ Open 2021;11:e047114. doi:10.1136/ bmjopen-2020-047114

- Prepublication history and additional supplemental material for this paper are available online. To view these files, please visit the journal online (http://dx.doi.org/10.1136/ bmjopen-2020-047114).

Received 21 November 2020

Revised 01 April 2021

Accepted 19 April 2021

Check for updates

(c) Author(s) (or their employer(s)) 2021. Re-use permitted under CC BY-NC. No commercial re-use. See rights and permissions. Published by BMJ.

${ }^{1}$ Florence Nightingale Faculty of Nursing, Midwifery \& Palliative Care, King's College London, London, UK

${ }^{2}$ Independent Service User and Carer Group, London, UK

${ }^{3}$ FOR WOMEN CIC, London, UK

Correspondence to

Sarah McAllister;

Sarah.McAllister@kcl.ac.uk

\section{ABSTRACT}

Objectives Our objectives were threefold: (1) describe a collaborative, theoretically driven approach to co-designing complex interventions; (2) demonstrate the implementation of this approach to share learning with others; and (3) develop a toolkit to enhance therapeutic engagement on acute mental health wards.

Design and participants We describe a theory-driven approach to co-designing an intervention by adapting and integrating Experience-based Co-design (EBCD) with the Behaviour Change Wheel (BCW). Our case study was informed by the results of a systematic integrative review and guided by this integrated approach. We undertook 80 hours of non-participant observations, and semistructured interviews with 14 service users ( 7 of which were filmed), 2 carers and 12 clinicians from the same acute ward. The facilitated intervention co-design process involved two feedback workshops, one joint co-design workshop and seven small co-design team meetings. Data analysis comprised the identification of touchpoints and use of the $\mathrm{BCW}$ and behaviour change technique taxonomy to inform intervention development.

Setting This study was conducted over 12 months at an acute mental health organisation in England.

Results The co-designed Let's Talk toolkit addressed four joint service user/clinician priorities for change: (1) improve communication with withdrawn people; (2) nurses to help service users help themselves; (3) nurses to feel confident when engaging with service users; (4) improving team relations and ward culture. Intervention functions included training, education, enablement, coercion and persuasion; 14 behaviour change techniques supported these functions. We detail how we implemented our integrated co-design-behaviour change approach with service users, carers and clinicians to develop a toolkit to improve nurse-patient therapeutic engagement.

Conclusions Our theory-driven approach enhanced both $E B C D$ and the BCW. It introduces a robust theoretical approach to guide intervention development within the codesign process and sets out how to meaningfully involve
Strengths and limitations of this study

- To our knowledge, our study is the first to combine and implement a new theory-driven co-designbehaviour change process with service users, carers and clinicians in a mental health setting.

- Our intervention development process was highly collaborative, with service users, carers and clinicians working together in equal and active partnership.

- Our process provided a systematic and replicable system for reporting the behavioural mechanisms of action behind our complex intervention toolkit.

- Although our process was highly collaborative, it was conducted at just one National Health Service site, which represents a possible limitation.

service users and other stakeholders when designing and implementing complex interventions.

\section{BACKGROUND}

Nurse-patient therapeutic engagement can broadly be described as the use of verbal and non-verbal interchange to improve a service user's mental health. ${ }^{12}$ Lack of highquality engagement on acute mental health wards is strongly associated with increased rates of self-harm, violence, aggression, absconding and poor perceptions of inpatient care. ${ }^{3}{ }^{4}$ Engagement may initiate and enhance the therapeutic relationship, ${ }^{5}$ which arguably has the greatest impact on treatment outcomes, over and above the specific interventions provided. ${ }^{67}$ However, nurses report high levels of acuity, reduced workforce, competing administrative duties and the nebulous nature of engagement as reasons for not engaging with service users. ${ }^{8-10}$ These 
factors also have a negative impact on nurses' job satisfaction, ${ }^{11}$ increasing the likelihood of burn-out and leaving the profession prematurely.

Reports from service users suggest that wards are experienced as devoid from warm, respectful therapeutic interactions. ${ }^{12}$ Pharmacological treatments are prioritised over collaborative clinician-patient engagement, which leaves service users feeling stigmatised and alienated from their care team. ${ }^{13}{ }^{14}$ Despite a recognition of the importance of collaborative care planning by clinicians, service users were often not involved in this process and felt as if they had no say in the trajectory of their care. ${ }^{15}$ Policymakers, researchers and patient advocacy groups globally have emphasised the importance of engagement in practice. ${ }^{16-18}$ However, lack of quality engagement is a long-standing, complex problem ${ }^{19} 20$ and few nursing interventions to improve engagement are reported in the literature.

One such intervention, predominantly implemented in the UK, is protected engagement time (PET). During PET, nurses devote a specified amount of time to regular engagement sessions with service users. ${ }^{21} 22$ PET originates from the refocusing model, which was a comprehensive series of interventions to improve inpatient services and reduce work strain on staff. ${ }^{23} 24$ The refocus model brought about improvements to the quality of care, staff sickness and costs, rates of absconding and selfharm. ${ }^{23}$ Following this, PET was adopted as a stand-alone intervention by mainstream policy (eg, ref 25), which resulted in its top-down implementation in many mental health services across England. Subsequent evaluations on both adult and older adult mental health wards found that while PET attempts to address nurses' opportunities to engage, it does not account for wider considerations about what is done within the engagement sessions. ${ }^{26-28}$ This may be because PET was intended to be used alongside other interventions, and its use as a stand-alone intervention stemmed from an atheoretical, common sense approach to implementation.

In response to PET's limitations, a Swedish study developed the Time to Talk (TT) intervention. ${ }^{29}$ TT is a form of PET, theoretically informed by two studies of everyday life on acute wards ${ }^{30}$ and the Tidal Model-a holistic model of nursing care that promotes the exploration of service users' own narratives. ${ }^{32}$ In a qualitative evaluation of $\mathrm{TT}^{33}$ service users reported that clinicians were more engaged after TT was implemented; however, their quantitative evaluation found no improvement in the quality of engagement as measured through the Caring Professional Scale. ${ }^{34}$ This mirrors evaluations of PET. ${ }^{26} 27$ Although PET and TT address nurses' opportunities to engage, they may not compensate for wider deficiencies in service provision such as poor supervision, clinical skills and personal motivations, ${ }^{2628}$ and neither were collaboratively developed with input from service users, carers and clinicians.

To better understand and enhance nurse-patient engagement in practice we previously conducted a systematic integrative review to develop a conceptual model of engagement. ${ }^{35}$ For high-quality engagement to occur, the model suggests that nurses must employ techniques that encompass five 'Principles of Engagement': (1) understand the person and their illness; (2) facilitate growth; (3) therapeutic use of self; (4) choose the right approach and; (5) emotional versus restrictive containment. The model drew on behaviour change theory ${ }^{36}$ to show that engagement is broadly influenced by both the service users' and nurses' capability, opportunity and motivation to engage. To address the limitations of previous interventions, we propose a collaborative, theory-driven approach to co-designing a complex intervention to improve the amount and quality of engagement on acute mental health wards. To do so, we have drawn from our model of engagement described above and adapted and integrated two existing approaches: Experience-based Co-design (EBCD) ${ }^{37} 38$ and the Behaviour Change Wheel (BCW). ${ }^{36}$

EBCD is a form of participatory action research which draws on user-centred design and user experience to improve healthcare services. ${ }^{37}$ The structured EBCD process, detailed in a freely available online toolkit, ${ }^{39}$ aims to meaningfully engage service users, carers and clinicians throughout a co-design process using observations, interviews and facilitated workshops. The BCW and accompanying behaviour change technique taxonomy version 1 (BCTTv1) has amalgamated 19 behaviour change theories to create a framework that guides intervention development. ${ }^{36}$ It follows three phases: (1) understand the behaviour; (2) identify intervention options; and (3) identify intervention content. At its core, the model suggests that capability, opportunity and motivation interact to create behaviours (COMB).$^{40}$ The Theoretical Domains Framework (TDF) ${ }^{41}$ is aligned in the model to the COM-B components and both are linked to nine intervention functions. The BCTTv1 is a taxonomy of 93 behaviour change techniques (BCTs). These 93 BCTs can be matched to the intervention functions to identify suitable BCTs, which make up the active ingredients of an intervention. ${ }^{42} 43$ Figure 1 maps the BCW phases, methods and tools to the phases, methods and tools of EBCD and provides a theory-driven basis for the co-design of behaviour change interventions.

Healthcare research and policy now recognise the importance of both co-designing interventions and using a robust theory to guide intervention development, ${ }^{44} 45$ but to date very few studies report on how to co-design complex healthcare interventions using a theory-driven approach. Currently, there are no published studies that develop interventions using EBCD informed by the $\mathrm{BCW}$. In response, we demonstrate the implementation of a theory-driven co-design-behaviour change process (figure 1) that was used to develop a complex intervention toolkit for promoting nurse-patient engagement on acute mental health wards.

We aim to: 
- Describe a collaborative, theoretically driven approach to co-designing complex interventions.

- Demonstrate the implementation of this process to share learning with others.

- Develop a toolkit to enhance therapeutic engagement on acute mental health wards.

\section{METHODS}

\section{Design}

This case study was guided by the UK Medical Research Council complex intervention framework ${ }^{46}$ and was theoretically driven by the content illustrated in figure 1 . The co-design process is reported in accordance with guidance for reporting intervention development studies in health research ${ }^{47}$ (online supplemental file 1). Participants gave written consent prior to being interviewed and again at the start of each co-design workshop. Posters that explained the purpose of the ward observations were displayed in common areas on the ward. Participation in observations was on an opt-out basis, to which nobody opted out.

\section{Setting}

The study was conducted with service users, carers and clinicians from one inner-London National Health Service (NHS) Foundation Trust in England, where the lead author had previously conducted exploratory work. ${ }^{5}$ The intervention ward has 18 beds and treats adults $^{18-64}$ experiencing an acute phase of severe

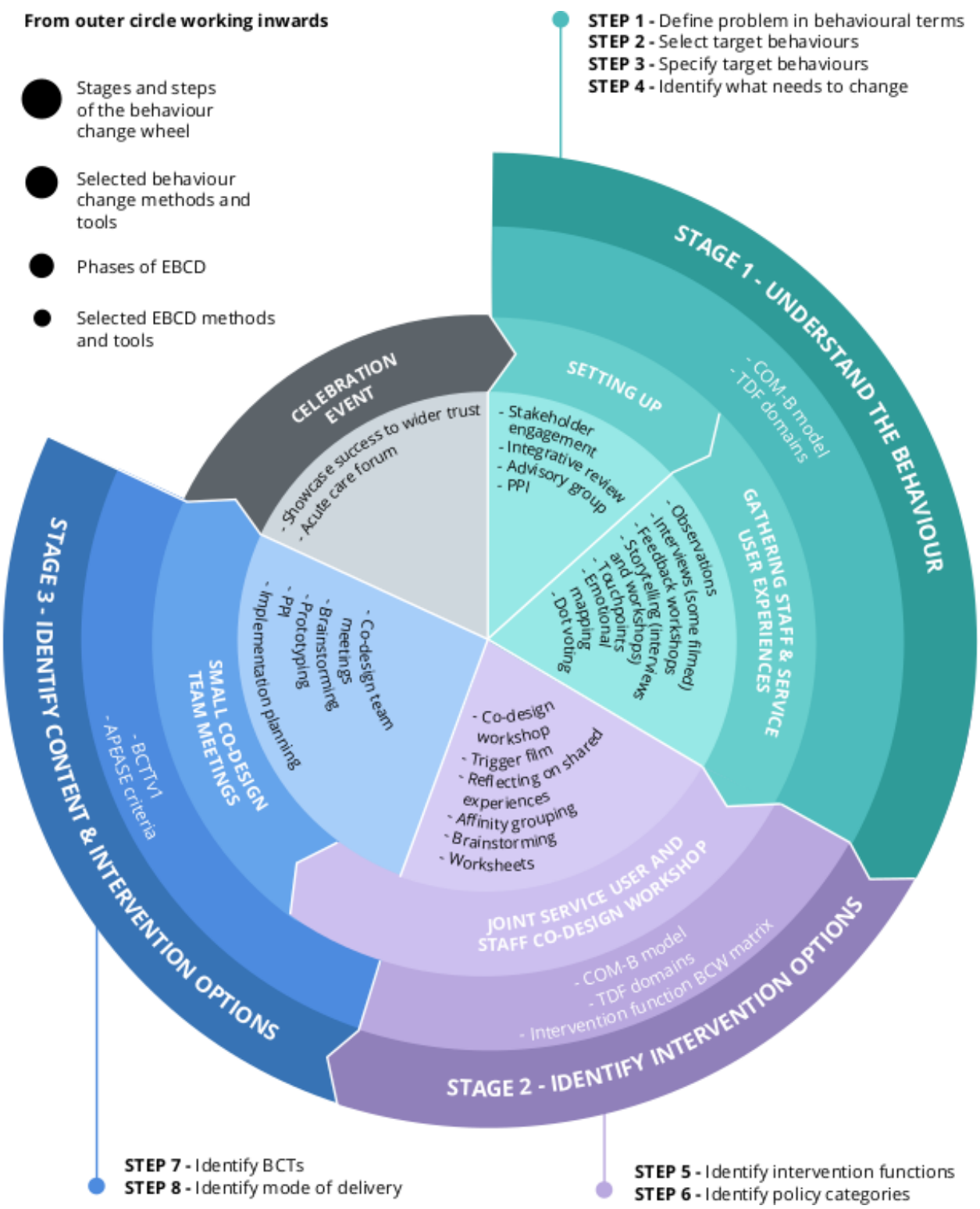

Figure 1 Integrated codesign-behaviour change model. APEASE, affordability, practicability, effectiveness/cost-effectiveness, acceptability, side effects/safety and equity; BCT, behaviour change technique; BCTTv1, behaviour change technique taxonomy version 1; BCW, behaviour change wheel; COM-B, capability, opportunity and motivation interact to create behaviours; $E B C D$, experience-based co-design; PPI, patient and public involvement; TDF, theoretical domains framework. 
mental illness. The ward is laid out along a corridor, with the nursing station and reception area at one end of the ward, the service user bedrooms running along both sides of the corridor and the service user lounge and day area at the opposite end of the ward to the nursing station. Service users are predominantly detained under the Mental Health Act. ${ }^{48}$ The ward consists of a multidisciplinary team of 20 clinicians, including 8 registered mental health nurses (RMNs), 7 healthcare assistants (HCAs), a peer support worker, an activities coordinator, an occupational therapist, a psychologist and a consultant psychiatrist. The nursing team works shift patterns from 07:30 to 21:30 or from 21:00 to 08:00 and all RMNs and HCAs are involved in direct patient care including care planning, oneto-one interactions and close and hourly observations. The RMNs are responsible for medication rounds. The ward provides timetabled daily activities run by the activities coordinator, and service users attend weekly ward rounds led by the consultant psychiatrist and an RMN. This project began in April 2018 and complemented other organisational improvement work to reimplement PET.
Participants

The co-design team was recruited through:

- A convenience sample of service users and carers via: (1) face-to-face contact and posters at community mental health teams (CMHTs) and (2) faceto-face contact and email at service user advocacy groups connected to the participating NHS organisation.

- A whole population sample of clinicians on the participating ward were invited to take part via presentations, posters, email and face-to-face meetings.

SM screened all potential participants, specifically looking for those who had, or had cared for somebody who had at least one inpatient admission at the organisation but was not currently experiencing a relapse. Eligible individuals were then guided through a written informed consent procedure. Figure 2 shows the recruitment process by type of participant and workshop attendance through the EBCD process. A total of 35 members were recruited to the co-design team including 15 service users, 2 carers, 10 RMNs,

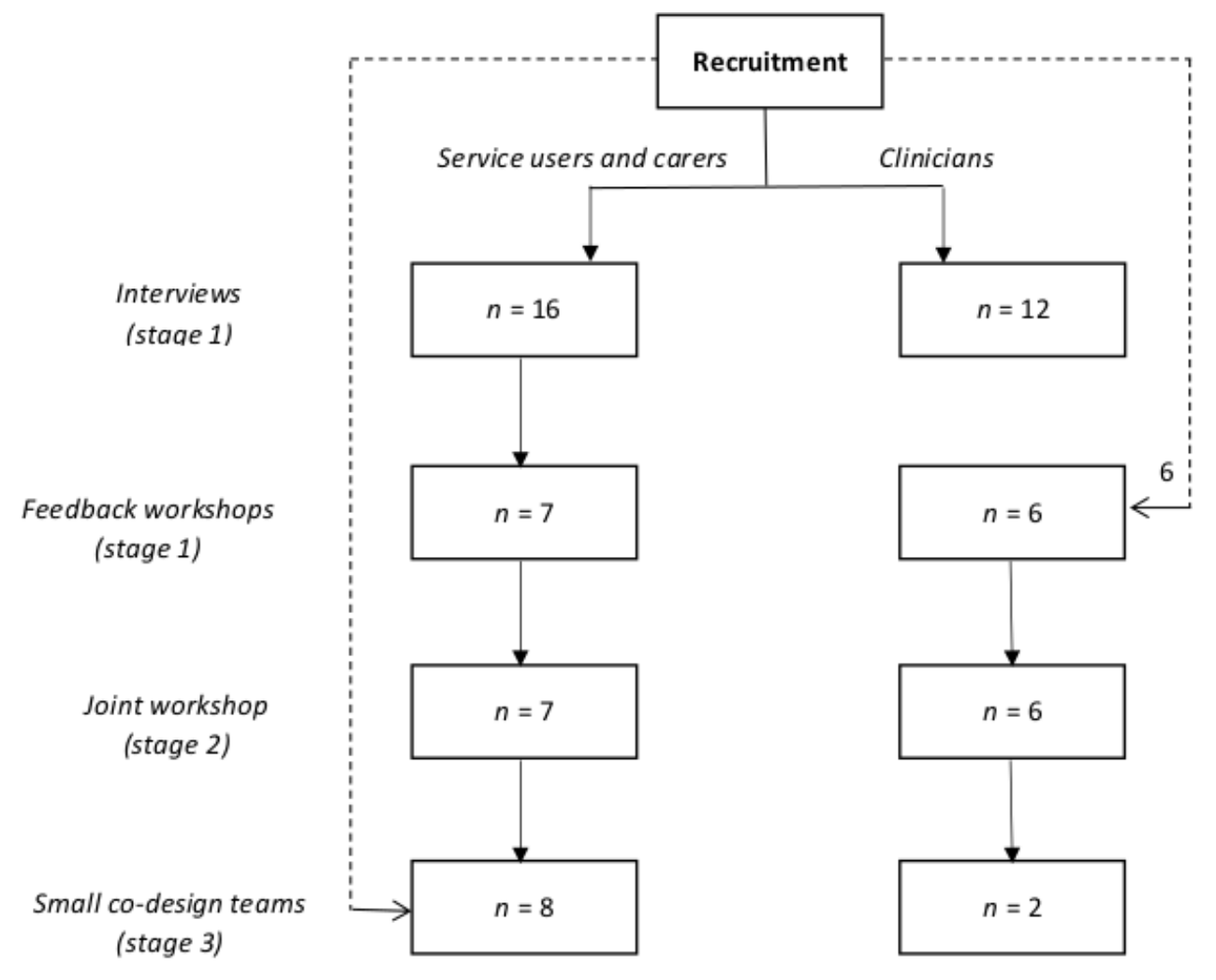

Service users and carers

Clinicians

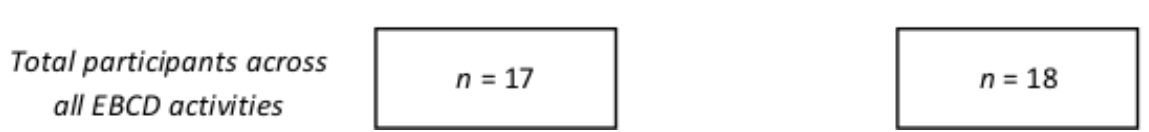

Figure 2 Recruitment process by type of participant and workshop attendance. EBCD, experience-based codesign. 
4 HCAs, 3 psychological therapy clinicians and 1 student nurse. Just over half of the codesign team were female (54\%) and just under half were from a black, Asian, and minority ethnic background (49\%). Participants' ages ranged from 18 to 64 years. Service users had a variety of mental illnesses, including psychotic disorders such as schizophrenia and bipolar affective disorder $(71 \%)$, personality disorder $(7 \%)$, anxiety $(7 \%)$ and eating disorder $(7 \%)$.

\section{Data collection and analysis}

Data collection methods and processes were aligned to the EBCD phases and BCW stages contained in figure 1 and informed by the aforementioned integrative review ${ }^{35}$ these included non-participant observations and semistructured interviews to gather service user, carer and clinician experiences, and feedback and co-design workshops to facilitate development of the engagement toolkit.

\section{Non-participant observations and semistructured interviews}

SM (a mental health nurse, previously unknown to the study participants and trained in the application of the EBCD and BCW approach) conducted 80 hours of nonparticipant observations on the acute ward between the hours of 07:30 and 15:00 or 13:30 and 21:30. Monday through Sunday. Observations were performed in 15 minute intervals, beginning with the first nurse encountered and continued until all nursing staff had been observed. Field notes were guided by Tyson and colleagues $^{49}$ and documented patterns of nurse-patient behaviour, nurse-patient dynamics, tone of voice, body language, potential influences on engagement and general ward atmosphere.

SM also interviewed 14 service users, 2 carers and 12 clinicians on a one-to-one basis at a location of their choice including university premises, offices at CMHTs or by telephone. All interviews were audio recorded and seven service user interviews were filmed in keeping with the EBCD approach. Interviews lasted between 30 and $80 \mathrm{~min}$. A topic guide was followed, informed by our review, ${ }^{35}$ the non-participant observations and the COM-B/TDF domains. ${ }^{41} 42$ Interviews addressed participants' experiences of engagement, barriers and facilitators to engagement, and clarified assumptions made from the observations.

Full details of the non-participant observations and semistructured interviews, including the inductive analysis of data to identify 'touchpoints' (emotionally significant points) of importance to the co-design team, are reported in a separate paper. ${ }^{50} \mathrm{~A}$ secondary deductive analysis of interview data, which is reported in this paper, was also undertaken to identify barriers to engagement. Deductive codes were based on the COM-B and TDF components of the BCW which were used as an a priori framework to analyse and thematically organise interview data. SM independently coded and themed the data using this framework. Extracts from both the filmed and audio-recorded interviews were also edited into a trigger film that was used to stimulate discussion at the feedback and co-design workshops.

\section{Feedback and codesign workshops}

Touchpoints and themes were shared at separate service user/carer and clinician feedback workshops and at a joint co-design team workshop. This ensured validity of the analysis, facilitated the joint selection of target behaviours based on the touchpoints and allowed intervention options and content to be agreed. Seven co-design team meetings were also established to work on specific priority areas. Consensus was reached through facilitated discussions and consensus building exercises including emotional mapping ${ }^{37}$ and affinity grouping. ${ }^{51}$

Input was also sought throughout the co-design process from two mental health patient and public involvement (PPI) groups based at the participating organisation. An advisory group consisted of a service user representative, one clinician and clinical academic experts in (A) the EBCD methodology and (B) therapeutic engagement, respectively. The service user representative cofacilitated the feedback workshops with SM who also facilitated the joint co-design and co-design team workshops with the assistance of another nurse researcher trained in the BCW approach. Three co-design team members wrote reflective accounts of their experiences of the co-design process and are coauthors of this paper.

\section{Patient and public involvement}

Service users and carers were at the heart of this research, being involved from conception, through execution and dissemination of this work.

\section{RESULTS}

Here we present our theory-driven approach to co-designing the Let's Talk complex intervention toolkit. Our findings are organised under the three stages (and eight constituent steps) of the BCW guide, as shown in figure 1.

\section{Stage 1: understanding the behaviours}

Step 1: define the problem in behavioural terms

Through previous research, ${ }^{5}$ our integrative review ${ }^{35}$ and initial discussions with our PPI, advisory groups and the clinical service lead, modern matron and divisional medical director at the NHS organisation, the behavioural problem was defined as the absence of highquality nurse-patient therapeutic engagement on acute mental health wards, that is, not using the Principles of Engagement identified in our review.

\section{Step 2: select target behaviour(s)}

In keeping with the EBCD methodology, it was important to understand how service users and staff typically experienced engagement prior to the identification of relevant areas for behavioural change. Through observations and semistructured interviews, the research team identified 28 touchpoints. Some examples of touchpoints were (1) I was left on my own and ignored; (2) my care 
Table 1 Service user and clinician priorities for change

\section{Service user priorities}

(1) Nurse-patient communication needs to be improved.

(2) Treat me like a human being.

(3) Forgive and forget.

(4) Help me help myself.

\section{Clinician priorities}

(1) Improve the way we communicate with service users.

(2) Improve the way that leave is communicated.

(3) Improve culture around response.

(4) Improve the way messages are handed over within the team. was robotic; and (3) as a nursing team we need to create better bonds with service users (full results found in ref $50)$.

To ensure credibility, the touchpoints were discussed during two facilitated feedback workshops-one for service users and one for clinicians. In an emotional mapping exercise, participants were encouraged to identify improvement priorities based on their touchpoints and assign associated behaviours (see online supplemental file 2 for breakdown of touchpoints into improvement priorities and associated behaviours). Participants then ranked their improvement priorities in a dot voting exercise and chose four priorities to take forward to the joint workshop (table 1). The service user and clinician priorities were as follows.

At the joint workshop, facilitated discussion encouraged participants to consider the potential impact, likelihood of change, spillover effect and ease of measurement of all the improvement priorities and associated behaviours. An affinity grouping exercise was conducted and through this, four shared improvement priorities were identified and agreed:

1. Improve communication with withdrawn people.

2. Nurses to help service users help themselves.

3. Increasing nurses' confidence when interacting with service users.

4. Improve team relations and ward culture.

\section{Step 3: specify target behaviour(s)}

EBCD focuses on identifying participants' improvement priorities as a way of bringing about change that is meaningful to service users, carers and clinicians. ${ }^{39}$ We used the BCW to examine each of the four joint improvement priorities. At the joint workshop, the co-design team formed into smaller groups with equal numbers of service users and clinicians. Each group completed a written exercise where they examined the joint priorities and associated behaviours in terms of who needs to perform the behaviour, what the person needs to do differently to achieve change and when, where and with whom they will do it (table 2). (See online supplemental file 3 for example of written exercise.)

\section{Step 4: identify what needs to change}

From our review and semistructured interviews with service users, carers and clinicians, the research team identified 26 barriers to engagement and mapped them to the COM-B/TDF domains. The barriers were discussed with participants at the feedback workshops to ensure credibility. At the joint workshop participants matched the barriers to their four joint improvement priorities. The barriers related to each COM-B component are discussed below, with the corresponding TDF domains presented in parentheses.

Table 2 Specification of behaviours for joint improvement priorities

\begin{tabular}{lllll}
\hline \multirow{2}{*}{$\begin{array}{l}\text { Joint improvement } \\
\text { priorities }\end{array}$} & Behaviour specification & & \\
\cline { 2 - 5 } $\begin{array}{l}\text { Improve communication } \\
\text { with withdrawn people. }\end{array}$ & $\begin{array}{l}\text { (1) Recognise who needs to engage; (2) } \\
\text { Respond in a timely and appropriate manner } \\
\text { when engaging. }\end{array}$ & Nurses & Acute ward & When service users \\
$\begin{array}{l}\text { Nurses to help service } \\
\text { users help themselves. }\end{array}$ & $\begin{array}{l}\text { (1) Give practical advice; (2) explain the } \\
\text { purpose of admission; (3) understand } \\
\text { the person; (4) facilitate growth; (5) give } \\
\text { discharge support. }\end{array}$ & Nurses & Acute ward & During service user's \\
& & & admission to an acute \\
ward.
\end{tabular}

\begin{tabular}{|c|c|c|c|c|}
\hline $\begin{array}{l}\text { Nurses must feel confident } \\
\text { when engaging with service } \\
\text { users. }\end{array}$ & $\begin{array}{l}\text { (1) Have effective therapeutic conversations; } \\
\text { (2) Articulate practical procedures in an } \\
\text { understandable way; (3)Reduce anxiety when } \\
\text { engaging. }\end{array}$ & Nurses & Acute ward & $\begin{array}{l}\text { When engaging with a } \\
\text { service user. }\end{array}$ \\
\hline
\end{tabular}




\section{Capability}

Participants agreed that nurses often had limited knowledge and inadequate training in therapeutic engagement techniques (skills and knowledge):

Although I've been doing this for almost fiveyears it's like sometimes with certain patients you just don't know what to say...I wish there could be some training to understand that stuff.-RMN6

Nurses also felt that the very nature of having a mental health problem could make it difficult to engage, and while service users agreed that their mental illness and medication effects could negatively impact engagement (memory/attention/decision process), they were able to describe helpful engagement techniques that nurses could employ, even with the most acutely unwell people. This further highlighted the need to improve nurses' engagement skills:

Sometimes you have a lot more patients who are unwell or sometimes they're less unwell, so engagement fluctuates week on week from that point of view.-RMN2

\section{Opportunity}

It was felt that there needed to be a cultural shift on the ward and within the organisation so that nurse-patient engagement activities were supported and valued in the same way as other tasks such as hourly observations or administrative duties (social influences):

It was a numbers game, everyone's taking handover, another one's doing checks, some are on break...in an ideal world allocate friendly HCAs just to sit with patients.-SU7

There was unanimous agreement that lack of resources negatively impacted on nurses' ability to engage therapeutically:

The problem for me lies on the number of staff, that is not enough...-C1

This created an untherapeutic ward environment where 'professionals would run around like mad rabbits not giving any attention to the patients'.-SU2 (environmental contexts and resources)

\section{Motivation}

Nurses felt that they could not always trust all members of their team to carry out the job in the right way. This created a feeling of helplessness for some nurses, which deterred them from engaging therapeutically (beliefs about capabilities):

I became very aware that when there is an incident, I'm left on my own...I stopped trusting the team...I couldn't rely, therefore I needed to take a step back from the patients.-HCA2
Service users were also deterred from approaching nurses for engagement because they felt nurses often did not understand their problems or would punish them if they asked for therapeutic engagement too often (beliefs about consequences):

I kept myself to myself because even when I asked for simplest of things I was made to wait for ages so I would get frustrated, but if I showed frustration no doubt that would be on my notes and I would get set back.-SU4

As well as issues of trust, the ward staff felt as though their team were transient, with many long-standing nurses leaving to work elsewhere. This led to a lack of shared responsibility. Therapeutic engagement could easily 'fall through the cracks-HCA1, and when poor-quality engagement was witnessed, it was rarely followed up by a senior member of the team. This made some nurses feel they could not be bothered to engage:

I mean to put it blunt; I know it sounds really bad...I can't be bothered.-RMN5

There was also a blurring of professional roles, where although nurses knew they should engage, they left it to other professionals such as the occupational therapist or activities coordinator:

I can completely understand why nurses want separate roles because they would say you don't do our job so why should we do yours, but I do take people out on escorts and I do blur the boundaries there.-PT1.

When asked to give examples of nurse-patient engagement, many service users spoke about engagement with professionals other than nurses. This shows both the lack of engagement from nurses and the difficulty service users have in delineating between the nursing role and the role of other health professionals (social/professional identity).

There was a general sense from nurses that therapeutic engagement 'didn't always help people'-RMN8 (optimism). This led some nurses to feel anxious about engaging therapeutically, particularly when they felt they did not have the required skills. When this was coupled with feelings of frustration at the perceived lack of managerial support, nurses reported feeling drained, burnt out and demotivated (emotions):

One of the biggest problems is the management style which on paper, yes, it seems to be doing everything right, but in practice they have a very poor relationship with their staff and that does impact on performance...I just feel like no one cares about you, so why give up your time?-RMN3

Stage 2: identify intervention options

Step 5: identify intervention functions

PPI and advisory group meetings highlighted that some of the terminology used to describe intervention functions 
Table 3 Practical examples of behaviour change wheel functions given to co-design team

\section{Intervention function \\ Education (increase knowledge or understanding) \\ Persuasion (using communication to induce positive or negative feelings or stimulate action)}

Incentivisation (create an expectation of reward)

Coercion (create an expectation of punishment or cost)

Training (imparting skills)

Restriction (using rules to reduce/increase the opportunity to engage in target behaviour)

Environmental restructuring (changing physical or social
context)

Modelling (providing an example for people to aspire to or imitate)

Enablement (increasing means or reducing barriers to increase capability beyond environmental restructuring)

\section{Practical example given to co-design team}

Service users meet with nursing staff once they have recovered and describe their experiences while on the ward.*

Have a poster on the ward that shows people happily engaging, with a message that reminds clinicians that engagement is part of their job, it is not 'slacking off'. *

Offer a prize for the ward that has the best patient feedback regarding interactions. $\dagger$

At discharge, ask service users to provide feedback to the ward about the quality of interactions provided and hold staff accountable for this. *

Training programme that enables nurses to role-play with service users, so they gain skills on how to deal with service users' problems. *

Nurses stop paperwork/admin during mealtimes and sit with service users and have a cup of tea or some food.*

Give service users cards that display different emotions and if they want to talk they can put the card on their door so nurses know to approach them.*

Have a therapeutic engagement champion who promotes engagement and helps nurses to carry out group activities with patients.*

Have a ward diary for interactions that a member of staff is responsible for each shift.*

*Example that came from participant interviews.

†Example developed from behaviour change wheel (BCW) guide.

would not be suitable to use with our participants. Words such as 'coercion' can have negative connotations to mental health service users. Instead, practical examples that captured the essence of each intervention function were provided to participants at the joint co-design workshop. In a written exercise they were encouraged to use these examples to think about intervention functions that could address their four joint improvement priorities. Where possible we modelled these examples on illustrations from interviews with service users, carers and clinicians. Where this was not possible, we developed examples from the BCW book ${ }^{36}$ (table 3).

Participants identified five intervention functions that were relevant to bringing about the desired change. These were (1) training; (2) education; (3) enablement; (4) coercion; and (5) persuasion. Through discussions with senior management, the research team also identified restriction as a relevant function. The links between the COM-B/TDF domains and the intervention functions are shown in table 4 .

\section{Step 6: identify policy categories}

The BCW includes policy categories which may help to support the delivery of an intervention. Through discussion with senior management, the research team identified communication/marketing, guidelines and social planning as potentially relevant to facilitating our intervention. As such, the Principles of Engagement described in the introduction of this paper were included within Trust policy on therapeutic engagement and observations, and these principles will be directly linked with other components of the intervention, such as a training film described below.

\section{Stage 3: identifying intervention content and implementation options}

Steps 7 and 8: identify BCTs and mode of delivery

Rather than provide participants with a long list of BCTs, the written exercise at the joint workshop encouraged them to design intervention strategies they thought relevant to each of the four priorities and its influencing factors. The research team retrospectively assigned BCTs to the participants' examples and selected further BCTs and intervention strategies not identified during the joint workshop. These were the basis for the development of the first intervention prototype.

The prototype was further refined through an iterative process of email exchanges, telephone calls, a PPI meeting, seven small co-design team meetings and finally presentation of the work at an organisation-wide acute care forum. As per the BCW guide,$^{36}$ the affordability, practicability, effectiveness/cost-effectiveness, acceptability, side effects/safety and equity (APEASE) criteria were used in an adapted form (see online supplemental file 4) to stimulate discussion and ideas. These criteria ultimately informed the choice of intervention strategies for each improvement priority.

Fourteen BCTs were considered relevant to the Let's Talk intervention toolkit. Table 4 shows the link between each phase of the behaviour change intervention design process, the 14 BCTs and the intervention strategies and 


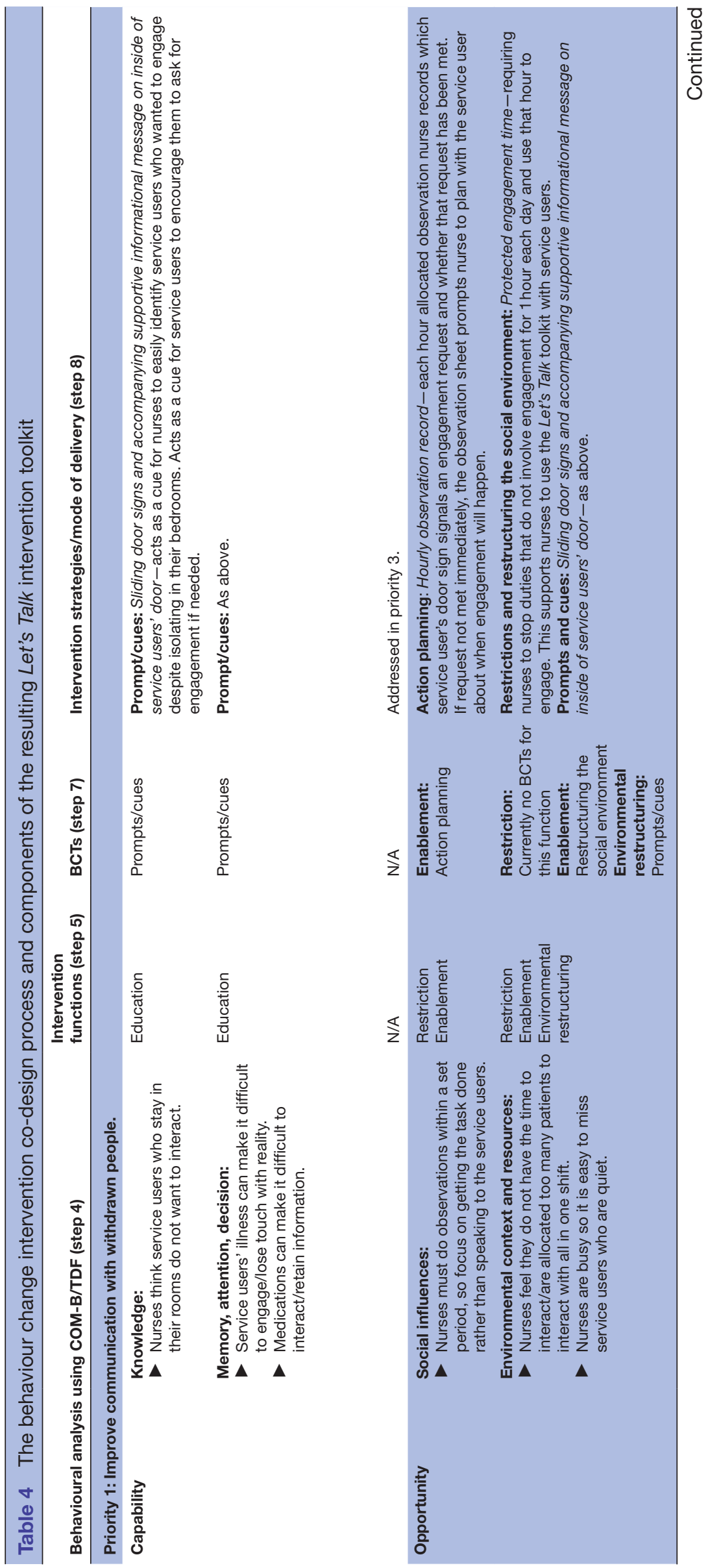

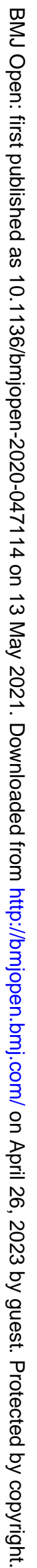




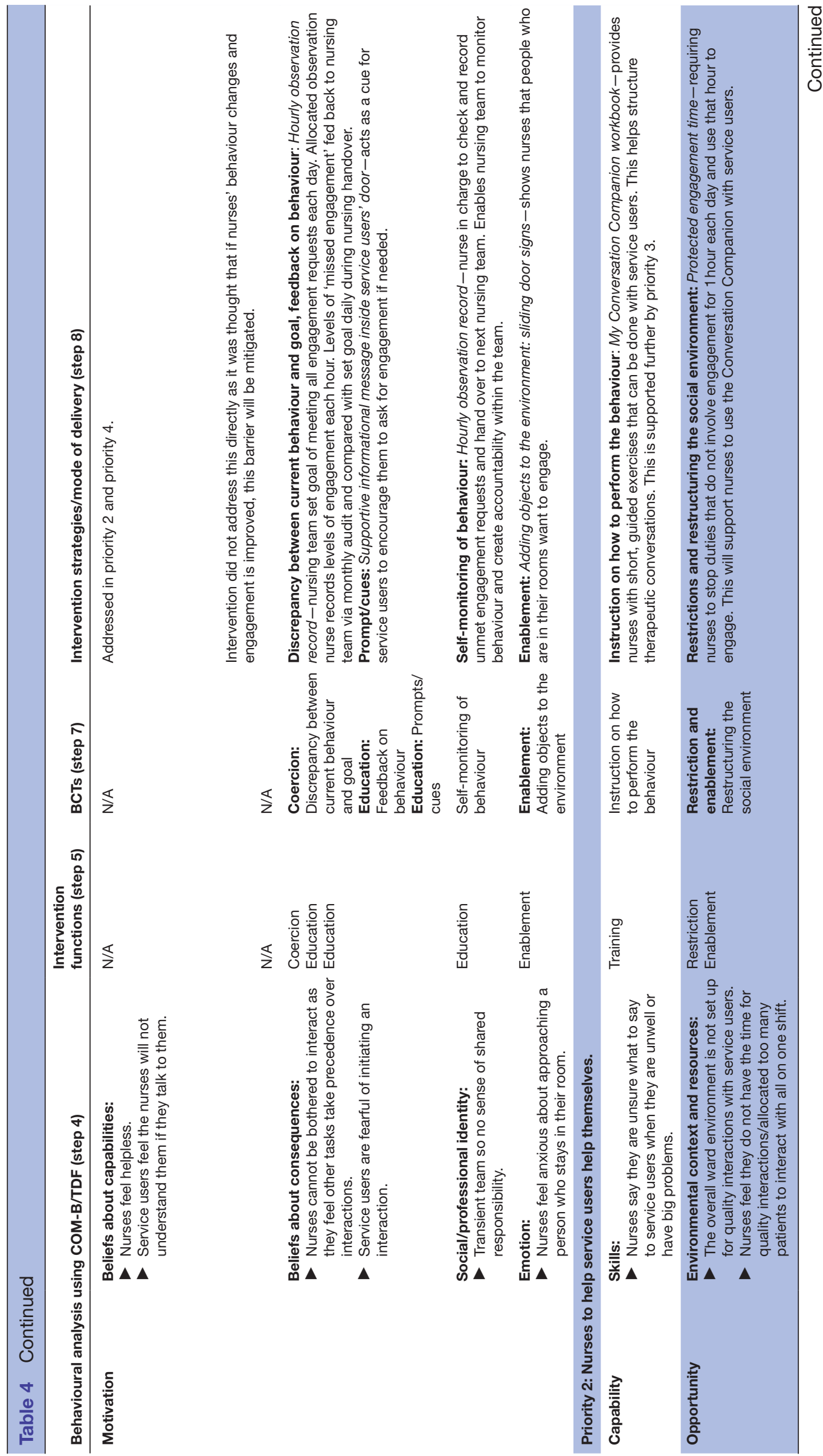

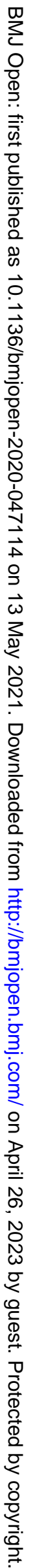




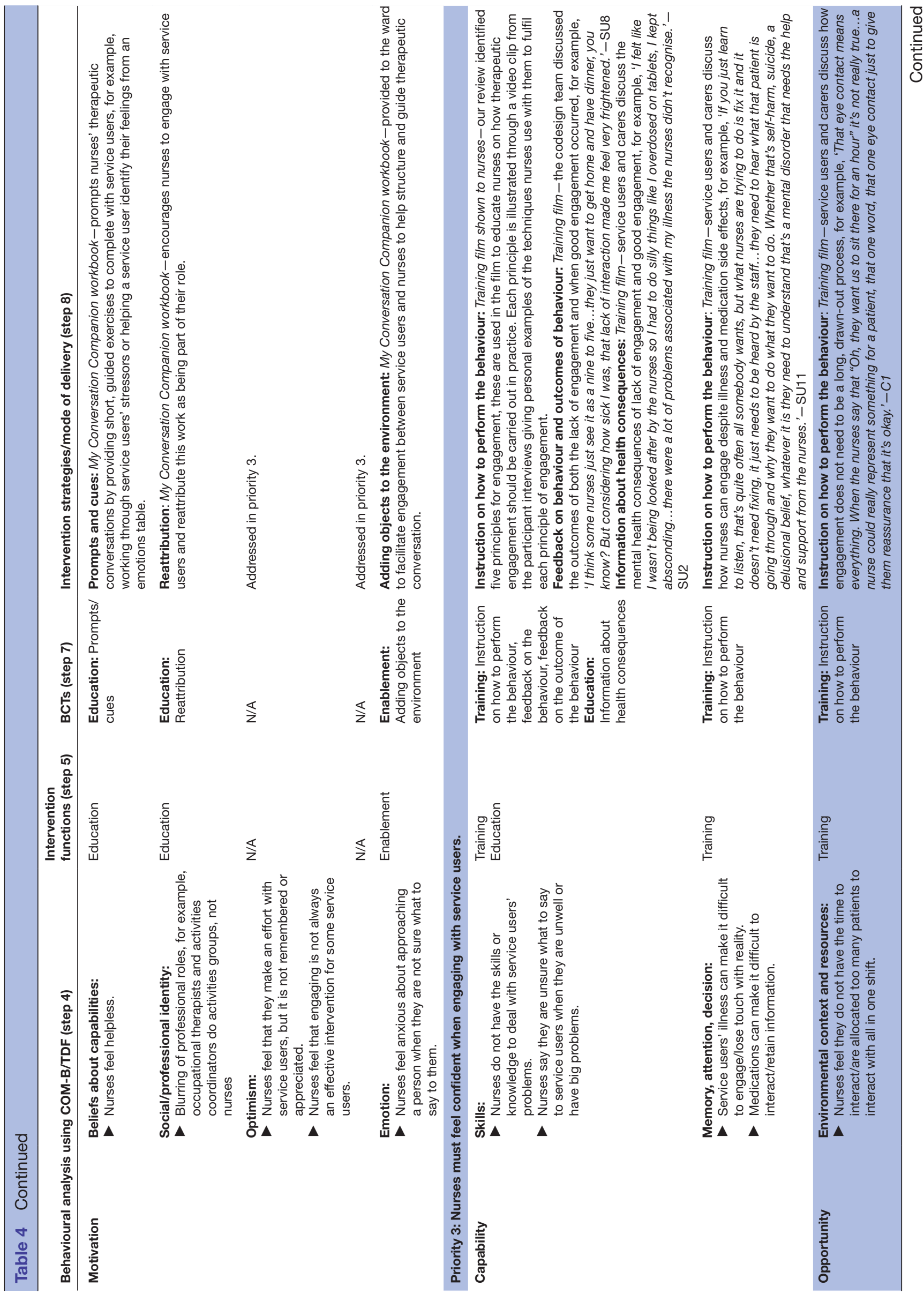




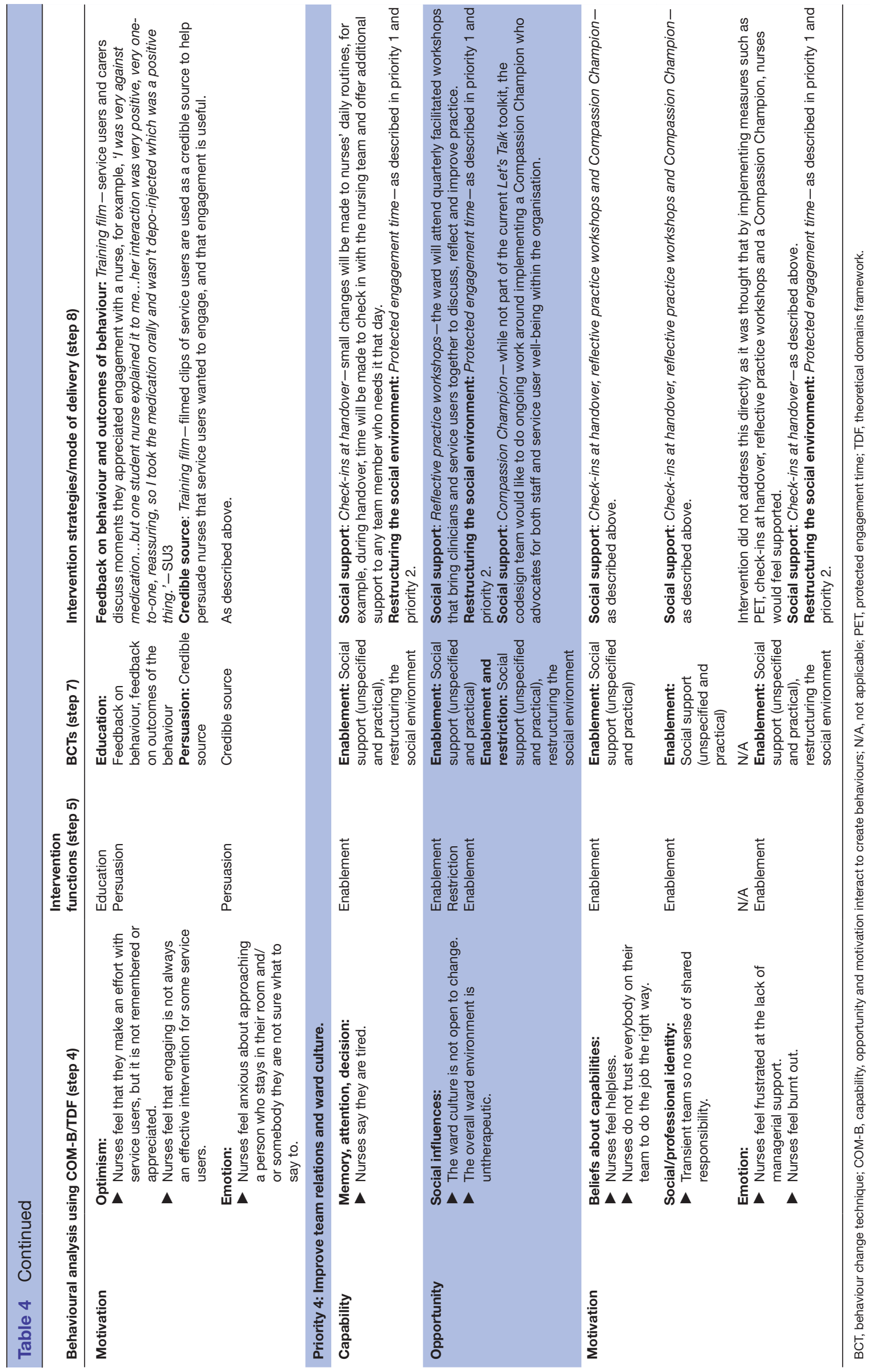


modes of delivery which resulted from the co-design process.

The Let's Talk toolkit consisted of four main components, linked to the co-design team's four joint improvement priorities:

1. A 30 minute training film for nurses, delivered by service users and carers to be shown to nurses at the start of the intervention. Service users and carers discuss good and bad engagement techniques and personal accounts of their experiences of engagement while an inpatient, structured by our model of engagement.

2. An illustrated workbook called My Conversation Companion which includes guided exercises that nurses and service users can do together to help structure therapeutic conversations.

3. Signs attached to the outside of service users' bedroom doors to enable them to indicate, with a sliding panel, whether they would like engagement time or not. The signs are linked to the hourly nursing observation record, where each hour nurses will be required to record if a service user has requested engagement and if that request has been fulfilled. 'Missed engagement' will be handed over at each nursing shift with the expectation that it is fulfilled that day. Observation records will be audited each month and feedback given to the nursing team. Additionally, an illustrated sign on the inside of service users' doors will encourage service users to use the signs if they want to engage.

4. Changes to nurses' daily routines, for example, during handover, time will be made to check in with the nursing team and offer additional support to any team member that needs it that day. Additionally, quarterly facilitated workshops will bring clinicians and service users together to discuss, reflect and improve practice. While conducting this work, the organisation was simultaneously discussing the potential addition of one extra staff member per shift. Our co-design team felt this would be beneficial to improving therapeutic engagement; however, a decision on this is yet to be made. Through discussions with the chief nurse, assistant director of nursing and divisional medical director and presentation of the work at an acute care forum it was agreed that the Let's Talk intervention would support the relaunched implementation of PET within the organisation. Discussion with participants revealed that they supported this and considered some form of PET essential to support nurses to use Let's Talk in practice. See online supplemental files 5 and 6 for the toolkit.

\section{DISCUSSION}

The delivery of high-quality nurse-patient therapeutic engagement is a complex issue that requires input from service users, carers, clinicians and researchers alike. Interventions to improve engagement must be multifaceted and encompass service users, carers and clinicians' capabilities, opportunities and motivations to engage. We used the methodical and evidence-based framework of the
BCW to guide intervention development within a co-design process. This enhanced the process by supporting its "intrinsically desirable qualities" ${ }^{52}$ with a robust theoretical underpinning that facilitated a full analysis of existing barriers and behaviours among its principal stakeholders. Although Larkin and colleagues ${ }^{53}$ suggest that it may be unrealistic to expect co-design participants to generate solutions to long-standing problems within a short space of time, supporting participants' ideas with a systematic and methodical theory of behaviour change may help mitigate that limitation.

Recent literature encourages a systematic, comprehensive and transparent approach to intervention development. ${ }^{40}$ However, many behaviour change interventions are poorly defined and do not use consistent language to describe their mechanisms of action ${ }^{55}$ making it difficult to pinpoint what did and did not work, which also reduces the ability to compare such interventions. ${ }^{41}$ The BCW enabled us to identify, understand and describe the mechanisms of action behind Let's Talk which is likely to both improve its effectiveness ${ }^{56}$ and enable us to review and refine intervention targets after preliminary testing. It also emphasised the importance of addressing nurses' capability, opportunity and motivation to engage. Previous interventions such as PET focus predominantly on the opportunities nurses have to engage, but do not consider whether a nurse may be capable or motivated to engage. This may explain why evaluations of PET have not shown improvements in the quality of engagement. ${ }^{57}$ To our knowledge, this is the first intervention aimed at improving engagement to be developed and presented in this comprehensive, systematic and transparent manner.

Although systematic, the BCW approach may be considered somewhat prescriptive. This can clash with the underlying principles of co-production and co-design, which demand democratic, innovative and creative techniques. ${ }^{58}{ }^{59}$ The concept of co-production in mental health was not commonplace even 5 years ago. ${ }^{60}$ Traditionally, professional knowledge had a higher status than service users' lived experiential knowledge. ${ }^{61} 62$ Despite some notable exceptions (eg, ref 63 64), service user participation in research was, and often still is, tokenistic, with participants having little influence over defining the problems or required changes. ${ }^{62}{ }^{65}$ It was essential that our process acknowledged, explored and addressed these power differentials so as not to reinforce these entrenched ideals.

Academic language and terminology can preserve power differentials and compromise user and clinician participation. ${ }^{667}$ People who suffer from mental health problems experience effects that can negatively impact cognition and concentration, often exacerbated by medications. ${ }^{68}$ The use of overly technical language may disproportionally affect people from this group and may lead to exclusion and disempowerment, ${ }^{69}$ which mirror some of the alienating experiences faced while an inpatient on acute wards (eg, ref 70). While the COM-B model uses relatively simple terminology, ${ }^{40}$ the language used to 
describe the intervention functions was particularly problematic. Intervention functions such as 'coercion' and 'restriction' may have triggered difficult emotions for some of our participants. These words describe negative ward experiences, for example, when clinicians coerce service users into taking medication, ${ }^{71}$ or when liberties are restricted due to treatment under the Mental Health Act $1983 .{ }^{48}$ This was also true of the clinicians who participated in our study. Suggesting that they lacked 'skills' or 'knowledge' was likely to alienate them from the process and make them feel devalued.

To ensure fidelity to the underlying principles of co-design we therefore tailored the BCW approach to the needs of the co-design team. The research team found that providing practical examples of each intervention function, using language from the service users, carers and clinicians' interviews, was a suitable way of adhering to the principles of co-design and using evidence-based theory in a non-alienating, confirmatory way. Although APEASE criteria were not considered to contain triggering terminology, some of the language was overly technical which also risked alienating co-design team members. The research team therefore translated the APEASE criteria into more accessible language. Furthermore, the co-design team were encouraged to design their own intervention content based on the behavioural analysis. The research team retrospectively assigned BCTs and confirmed these with the co-design team. This adhered to the underlying principles of co-design by foregrounding service user experience (rather than privileging academic knowledge over experiential knowledge), while also creating an intervention that could be clearly and methodically described through evidencebased theory and language.

Reflective accounts from three of our co-design team support the steps taken by the research team to ensure an inclusive, participatory process. While the potential for experiential reflections to trigger difficult emotions was anticipated, team members' expressed anxieties were soon 'quashed' by a 'safe and secure' environment in which members 'never felt pressured or judged'. This allowed the service users, carers and clinicians 'to support each other on an equal basis and share a common goal. The opportunity to share personal experiences emerged as an important dynamic across the three reflective accounts. It was variously described as 'a privilege', and an 'incredibly moving' and 'powerful' experience that allowed their expert knowledge to be used 'to implement new models of care and improve quality standards' that 'would make a real difference'. Consequently, these co-design team members described an 'enjoyable' and 'rewarding' process that engendered feelings of pride and empowerment. One member referred to it as a 'life changing' event that promoted self- esteem and self-awareness, and another reported the development of reflective skills. Notable also was the wider outreach and consultation that members undertook through liaison with professional colleagues, service users and carers in various institutional and community arenas, which mirrored their experience of the co-design process. This allowed stakeholders to express any concerns, ask questions and provide feedback. In turn, this led to "product refinements to make the workbook more accessible and easier to read'. As well as personal impact, these team members described interpersonal benefits including 'feeling (more) engaged with mental health professionals' and managing to 'engage in some really good work' with patients. They were also optimistic about the likely impact of this work moving forward. They sensed that service users and clinicians were 'inspired' by their work and believed the workbook would have "a ripple effect... and help create a cultural change within the organisation'. Each of the reflective accounts is provided in full in online supplemental file 7.

Beresford $^{72}$ argues that front-line clinicians can also be a marginalised group whose voices are often excluded. It is also vital to consider the needs of the service provider while embarking on participatory work. ${ }^{73}$ We implemented several facilitative measures such as providing backfill money so clinicians could attend the feedback and joint workshops and offered flexibility with participation in the small co-design teamwork, for example, emailing instead of face-to-face meetings and piggybacking staff meetings. However, enabling clinicians to participate equally was challenging. Unfortunately, organisational structures such as shift patterns and staffing levels impacted on clinicians' ability to fully participate. Regular staff meetings or reflective practice groups were also not in place. When given the opportunity to participate, clinicians were motivated, and meaningful participation was possible during the feedback and joint workshops. However, without organisational support structures to provide clinicians time to undertake the ongoing co-design work, much of the prototyping and iterative development of the intervention components were undertaken by the service users. This is a common issue evident in co-design studies in both mental health and general settings. ${ }^{74}$ There is a need for healthcare organisations to reconfigure their services so clinicians can meaningfully participate in such endeavours and encourage a sense of joint ownership over the work.

Although the process was highly collaborative and involved service users, carers and clinicians to varying degrees, it was conducted at just one NHS site, which represents a possible limitation. Transferability of our processes to other settings cannot be guaranteed. However, to our knowledge, this is the first time the BCW has been translated for use with participants who have mental health problems and used within an integrated co-design-behaviour change process. This new and novel approach will require further testing to ascertain whether it is suitable and translatable to other intervention development processes. Given that participants were a selfselecting, motivated sample of clinicians, service users and carers, their views may not be representative of all patients and clinicians in the organisation. During the final stage of co-designing Let's Talk the global COVID-19 pandemic took place. We continued our co-design 
activities remotely; however, a planned quasiexperimental pretest/post-test using a structured observational tool ${ }^{49}$ had to be postponed. The tool examines the amount, type (eg, interactive, individual, verbal, non-verbal or solitary) and quality (eg, positive feedback, praise, smile, ignoring, reprimand, discouragement, neutral behaviours) of nurse-patient interactions. ${ }^{49}$ Pretest data on one control and one intervention ward were collected in April to June 2019 and we plan to collect post-test data when we are able to do so and publish the results of this study.

\section{CONCLUSIONS}

This paper has described the implementation of a new theory-driven co-design-behaviour change approach used to develop the Let's Talk intervention toolkit. It offers tools that others may use, or adapt as necessary, to implement the approach in their settings. It also describes the behavioural mechanisms behind the Let's Talk intervention toolkit to improve the amount and quality of nursepatient therapeutic engagement on acute mental health wards. Our paper makes a timely and novel contribution to further both participatory methods and behaviour change theory. The approach enhances EBCD by introducing a robust behavioural change theory to help guide the development of a complex intervention. In turn, our participatory approach also enhances the BCW by setting out a practical guide on how to meaningfully involve service users and other stakeholders when designing complex implementation interventions.

Twitter Sarah McAllister @SarahMc_RMN, Alan Simpson @cityalan and Glenn Robert @gbrgsy

Acknowledgements The authors thank all the service users, carers and clinicians who gave their time to the codesign process. The authors also thank lain Ryrie, publication coach at King's College London, for his assistance with early drafts of this paper. And, finally, thank you to Soak Digital for designing figure 1, Peter Moorey for his illustrations as part of the Let's Talk toolkit and loanna Xenophontes and Sarah Combes for cofacilitating the EBCD workshops.

Contributors SM conceived the study, secured the research funding, facilitated the codesign process, analysed the data, contributed to designing intervention components and wrote the manuscript. GR, AS and VT participated in the design and coordination of the study, contributed to the analysis and helped draft the manuscript. NC, VDM and CS participated in the codesign process, contributed to designing intervention components and wrote reflective accounts for the manuscript. All authors read and approved the final manuscript.

Funding This report is independent research supported by the National Institute for Health Research (HEE/NIHR ICA Programme Clinical Doctoral Research Fellowship, Ms Sarah McAllister, ICA-CDRF2017-03-034). The views expressed in this publication are those of the author(s) and not necessarily those of the NHS, the National Institute for Health Research, or the Department of Health and Social Care.

Disclaimer The views expressed in this publication are those of the author(s) and not necessarily those of the NHS, the National Institute for Health Research or the Department of Health and Social Care.

Competing interests None declared.

Patient consent for publication Not required.

Ethics approval Ethical approval for the study was obtained from the London Fulham Research Ethics Committee (reference: 18/L0/2193).

Provenance and peer review Not commissioned; externally peer reviewed.

Data availability statement Data are available upon reasonable request. All data relevant to the study are included in the article or uploaded as supplementary information. All data generated or analysed during this study are included in this published article and its accompanying supplemental information files.

Supplemental material This content has been supplied by the author(s). It has not been vetted by BMJ Publishing Group Limited (BMJ) and may not have been peer-reviewed. Any opinions or recommendations discussed are solely those of the author(s) and are not endorsed by BMJ. BMJ disclaims all liability and responsibility arising from any reliance placed on the content. Where the content includes any translated material, BMJ does not warrant the accuracy and reliability of the translations (including but not limited to local regulations, clinical guidelines, terminology, drug names and drug dosages), and is not responsible for any error and/or omissions arising from translation and adaptation or otherwise.

Open access This is an open access article distributed in accordance with the Creative Commons Attribution Non Commercial (CC BY-NC 4.0) license, which permits others to distribute, remix, adapt, build upon this work non-commercially, and license their derivative works on different terms, provided the original work is properly cited, appropriate credit is given, any changes made indicated, and the use is non-commercial. See: http://creativecommons.org/licenses/by-nc/4.0/.

\section{ORCID iDs}

Sarah McAllister http://orcid.org/0000-0002-0448-006X

Glenn Robert http://orcid.org/0000-0001-8781-6675

\section{REFERENCES}

1 Cormack D. Psychiatric nursing observed. London: Royal College of Nursing, 1976.

2 Evans EC. Exploring the nuances of nurse-patient interaction through concept analysis: impact on patient satisfaction. Nurs Sci $Q$ 2016;29:62-70

3 Papadopoulos C, Ross J, Stewart D, et al. The antecedents of violence and aggression within psychiatric in-patient settings. Acta Psychiatr Scand 2012;125:425-39.

4 Wykes T, Csipke E, Williams $\mathrm{P}$, et al. Improving patient experiences of mental health inpatient care: a randomised controlled trial. Psychol Med 2018;48:488-97.

5 McAllister S, McCrae N. The therapeutic role of mental health nurses in psychiatric intensive care: a mixed-methods investigation in an inner-city mental health service. J Psychiatr Ment Health Nurs 2017;24:491-2.

6 Duncan BL, Miller SD, Wampold BE, et al. The heart and soul of change: delivering what works in therapy. Washington: American Psychological Association, 2010.

7 Priebe S, Mccabe R. Therapeutic relationships in psychiatry: the basis of therapy or therapy in itself? Int Rev Psychiatry 2008;20:521-6.

8 Seed MS, Torkelson DJ, Alnatour R. The role of the inpatient psychiatric nurse and its effect on job satisfaction. Issues Ment Health Nurs 2010;31:160-70.

9 Care Quality Commission. The state of care in mental health services 2014 to 2017: Findings from CQC's programme of comprehensive inspections of specialist mental health services, 2017. Available: https ://www.cqc.org.uk/sites/ default/files/ 20170 720_state ofmh report.pdf [Accessed 23 Oct 2020].

10 Moreno-Poyato AR, Montesó-Curto P, Delgado-Hito P, et al. The therapeutic relationship in inpatient psychiatric care: a narrative review of the perspective of nurses and patients. Arch Psychiatr Nurs 2016;30:782-7.

11 Moreno-Poyato AR, Delgado-Hito P, Suárez-Pérez R, et al. Improving the therapeutic relationship in inpatient psychiatric care: assessment of the therapeutic alliance and empathy after implementing evidencebased practices resulting from participatory action research. Perspect Psychiatr Care 2018;54:300-8.

12 Cutcliffe JR, Santos JC, Kozel B, et al. Raiders of the lost art: a review of published evaluations of inpatient mental health care experiences emanating from the United Kingdom, Portugal, Canada, Switzerland, Germany and Australia. Int J Ment Health Nurs 2015;24:375-85.

13 Beresford P, Perring R, Nettle M, et al. From mental illness to a social model of madness and distress. London: Shaping Our Lives, 2016. www.shapingourlives.org.uk

14 McKeown M, Wright K, Mercer D. Care planning: a neoliberal three card trick. J Psychiatr Ment Health Nurs 2017;24:451-60.

15 Coffey M, Hannigan B, Barlow S, et al. Recovery-focused mental health care planning and co-ordination in acute inpatient mental health settings: a cross national comparative mixed methods study. BMC Psychiatry 2019;19:115. 
16 Beresford P. Beyond the usual suspects, towards inclusive user involvement in shaping our Lives/INVOLVE, 2013. Available: https:// www.shapingourlives.org.uk/documents/BTUSReport.pdf [Accessed 09 Jun 2020].

17 Department of Health. From values to action: The Chief Nursing Officer's review of mental health nursing. London, 2006.

18 World Health Organization. Mental health action plan for 2013-20205. World Health Organisation, 2013.

19 Altschul A. Patient/Nurse interaction: a case study of interaction patterns in acute psychiatric wards. Edinburgh: Churchill Livingston, 1972.

20 Sharac J, McCrone P, Sabes-Figuera R, et al. Nurse and patient activities and interaction on psychiatric inpatients wards: a literature review. Int J Nurs Stud 2010;47:909-17.

21 The King's Fund: Partnership CSI. The acute care collaborative. London, 2005.

22 Nolan FM, Fox C, Cheston R, et al. A feasibility study comparing UK older adult mental health inpatient wards which use protected engagement time with other wards which do not: study protocol. Pilot Feasibility Stud 2016;2:7.

23 Dodds P, Bowles N. Dismantling formal observation and refocusing nursing activity in acute inpatient psychiatry: a case study. $J$ Psychiatr Ment Health Nurs 2001;8:183-8.

24 Bowles N, Howard R. The refocusing model: a means of realising the National acute inpatient strategy. Mental Health Review Journal 2003;8:27-31.

25 Mental Health Act Commission. Risk, rights and recovery: the mental health act Commission twelfth biennial report 2005-2007. London: MHAC, 2008.

26 Edwards K. Evaluating protected time in mental health acute care. Nursing Times 2008:104:28-9.

27 Thomson LDA, Hamilton R. Attitudes of mental health staff to protected therapeutic time in adult psychiatric wards. $J$ Psychiatr Ment Health Nurs 2012;19:911-5.

28 Dodd E, Cheston R, Procter C, et al. Protected engagement time on older adult mental health wards: a thematic analysis of the views of patients, carers, and staff. Int J Ment Health Nurs 2018;27:608-18.

29 Molin J, Lindgren BM, Graneheim UH, et al. Does 'Time Together' increase quality of interaction and decrease stress? A study protocol of a multisite nursing intervention in psychiatric inpatient care, using a mixed method approach. British Medical Journal Open 2017;7:e015677.

30 Molin J, Graneheim UH, Lindgren B-M. Quality of interactions influences everyday life in psychiatric inpatient care-patients' perspectives. Int J Qual Stud Health Well-being 2016;11:29897-97.

31 Molin J, Graneheim UH, Ringnér A, et al. From ideals to resignation - interprofessional teams perspectives on everyday life processes in psychiatric inpatient care. J Psychiatr Ment Health Nurs 2016;23:595-04.

32 Barker PJ, Buchanan-Barker P. The tidal model: a guide for mental health professionals. London: Brunner-Routledge, 2005.

33 Molin J, Graneheim UH, Ringnér A, et al. Patients' experiences of taking part in Time Together - A nursing intervention in psychiatric inpatient care. Int J Ment Health Nurs 2019;28:551-9.

34 Swanson KM. Predicting depressive symptoms after miscarriage: a path analysis based on the Lazarus paradigm. $J$ Womens Health Gend Based Med 2000;9:191-6.

35 McAllister S, Robert G, Tsianakas V, et al. Conceptualising nursepatient therapeutic engagement on acute mental health wards: an integrative review. Int J Nurs Stud 2019;93:106-18.

36 Michie S, Atkins L, West R. The behaviour change wheel: a guide to designing interventions. London: Silverback publishing, 2014.

37 Bate P, Robert G. Bringing user experience to healthcare improvement: the concepts, methods and practices of experiencebased design. Radcliffe Publishing: Oxford, 2007.

38 Robert G, Cornwell J, Locock L, et al. Patients and staff as codesigners of healthcare services. BMJ 2015;350:g7714.

39 Point of Care Foundation. Experience-Based Co-design toolkit, 2020. Available: https://www.pointofcarefoundation.org.uk/resource/ experience-based-co-design-ebcd-toolkit/ [Accessed 23 Oct 2020].

40 Michie S, van Stralen MM, West R. The behaviour change wheel: a new method for characterising and designing behaviour change interventions. Implement Sci 2011;6:42.

41 Cane J, O'Connor D, Michie S. Validation of the theoretical domains framework for use in behaviour change and implementation research Implement Sci 2012;7:37.

42 Michie S, Webb TL, Sniehotta FF. The importance of making explicit links between theoretical constructs and behaviour change techniques. Addiction 2010;105:1897-8.

43 Michie S, Richardson M, Johnston M, et al. The behavior change technique taxonomy (V1) of 93 hierarchically clustered techniques: building an international consensus for the reporting of behavior change interventions. Ann Behav Med 2013;46:81-95.

44 National Institute for Health and Care Excellence. Patient Carer \& Public Involvement \& Engagement, 2015. Available: https:// www.nice.org.uk/media/default/About/NICE-Communities/Publicinvolvement/Public-involvement-programme/PIP-process-guide-apr2015.pdf [Accessed 23 Oct 2020].

45 O'Cathain A, Croot L, Sworn K, et al. Taxonomy of approaches to developing interventions to improve health: a systematic methods overview. Pilot Feasibility Stud 2019;5:41

46 Craig P, Dieppe P, Macintyre S, et al. Developing and evaluating complex interventions: the new medical Research Council guidance. BMJ 2008;337:a1655.

47 Duncan E, O'Cathain A, Rousseau N, et al. Guidance for reporting intervention development studies in health research (guided): an evidence-based consensus study. BMJ Open 2020;10:e033516.

48 Mental health act: the mental health act 1983, 1983. Available: https://www.legislation.gov.uk/ukpga/1983/20/contents [Accessed 23 Oct 2020].

49 Tyson GA, Lambert WG, Beattie L. The quality of psychiatric nurses' interactions with patients: an observational study. Int J Nurs Stud 1995;32:49-58.

50 McAllister S, Simpson A, Tsianakas V, et al. "What matters to me": A multi-method qualitative study exploring service users', carers' and clinicians' needs and experiences of therapeutic engagement on acute mental health wards. Int J Ment Health Nurs 2021. doi:10.1111/inm.12835. [Epub ahead of print: 18 Jan 2021].

51 Dorrington P, Wilkinson C, Tasker L, et al. User-Centered design method for the design of assistive switch devices to improve user experience, accessibility, and independence. Journal of Usability Studies 2016;11:66-82.

52 Robert G, Locock L, Williams O. Co-producing and co-designing healthcare services and interventions. In: Dixon-Woods M, Martin G, eds. Great big book of improving quality and safety in healthcare. Cambridge University Press; The Healthcare Improvement Studies Institute, University of Cambridge: forthcoming, 2019.

53 Larkin M, Boden ZVR, Newton E. On the brink of genuinely collaborative care: experience-based Co-design in mental health. Qual Health Res 2015;25:1463-76.

54 Hoffmann TC, Glasziou PP, Boutron I, Milnes R, et al. Better reporting of interventions: template for intervention description and replication (TIDieR) checklist and guide. BMJ 2014;348:g1687.

55 Michie S, Carey RN, Johnston M, et al. From Theory-Inspired to theory-based interventions: a protocol for developing and testing a methodology for linking behaviour change techniques to theoretical mechanisms of action. Ann Behav Med 2018;52:501-12.

56 Michie S, Wood CE, Johnston M, et al. Behaviour change techniques: the development and evaluation of a taxonomic method for reporting and describing behaviour change interventions (a suite of five studies involving consensus methods, randomised controlled trials and analysis of qualitative data). Health Technol Assess 2015;19:1-188.

57 Molin J, Lindgren B-M, Graneheim UH, et al. Time together: a nursing intervention in psychiatric inpatient care: feasibility and effects. Int $J$ Ment Health Nurs 2018;27:1698-708.

58 Verschuere B, Vanleene D, Steen T. Democratic Co-Production: Concepts and Determinants. In: Brandsen T, Verschuere B, Steen T, eds. Co-Production and co-creation. London: Routledge, 2018: 243-51.

59 Farr M. Power dynamics and collaborative mechanisms in coproduction and co-design processes. Critical Social Policy 2018:38:623-44.

60 Gordon S, O'Brien AJ. Co-production: power, problems and possibilities international. Journal of Mental Health Nursing 2018;27:1201-3.

61 Boxall K, Beresford P. Service user research in social work and disability studies in the United Kingdom. Disability \& Society 2013;28:587-00.

62 Rose D, Kalathil J. Power, privledge and knowledge: the untenable promise of co-production in mental "health". Frontiers of Sociology 2019;4.

63 Simpson A, Jones J, Barlow S, et al. Adding sugar: service user and carer collaboration in mental health nursing research. $J$ Psychosoc Nurs Ment Health Serv 2014;52:22-30.

64 Bowers L, James K, Quirk A, et al. Reducing conflict and containment rates on acute psychiatric wards: the Safewards cluster randomised controlled trial. Int J Nurs Stud 2015;52:1412-22.

65 Rose D, Fleischmann P, Tonkiss F, et al. User and carer involvement in change management in a mental health context: review of the literature - report to the National Co-ordinating Centre for NHS Service Delivery and Organisation R\&D. London: NCCSDO, 2003. 
66 Rose D, Barnes M, Crawford M, et al. How do managers and leaders in the National health service and social care respond to service user involvement in mental health services in both its traditional and emergent forms? the ENSUE study. Health Services Delivery Research 2014;2.

67 Unertl KM, Fair AM, Favours JS, et al. Clinicians' perspectives on and interest in participating in a clinical data research network across the southeastern United States. BMC Health Serv Res 2018;18:568.

68 Yeisen RAH, Bjornestad J, Joa I, et al. Experiences of antipsychotic use in patients with early psychosis: a two-year follow-up study. BMC Psychiatry 2017;17:299.

69 Cahn E. Foreword: A commentary from the United States. In: Stephens L, Ryan-Collins J, Boyle D, eds. Co-production: a manifesto for growing the core economy. London: New Economics Foundation, 2008
70 Staniszewska S, Mockford C, Chadburn G, et al. Experiences of in-patient mental health services: systematic review. Br J Psychiatry 2019;214:1-10.

71 Sampogna G, Luciano M, Del Vecchio V, et al. Perceived coercion among patients admitted in psychiatric wards: Italian results of the EUNOMIA study. Front Psychiatry 2019;10:316.

72 Beresford P. Why inclusive participation matters. Politics of Participation in Mental Health Seminar Proceedings, 2003.

73 Karlsen E, Sagvaag H. Keys to unlocking service provider engagement in constrained co-production partnerships. Action Research 2020;0:1-19.

74 Donetto S, Tsianakas V, Robert G. Using experience-based Codesign $(E B C D)$ to improve the quality of healthcare: mapping where we are now and establishing future directions. National Nursing Research Unit, King's College London, 2014. 